\title{
Facteurs associés à l'exposition élevée aux médicaments psychotropes identifiés lors d'une étude épidémiologique montréalaise
}

\section{Factors associated with high exposure to psychotropic drugs in a Montreal catchment epidemiological area}

\author{
Michel Perreault, Djemaâ-Samia Mechakra-Tahiri, Marie-Josée Fleury, El Hadj \\ Touré, Emma Mitchell et Jean Caron
}

Volume 38, numéro 1, printemps 2013

URI : https://id.erudit.org/iderudit/1019196ar

DOI : https://doi.org/10.7202/1019196ar

\section{Aller au sommaire du numéro}

\section{Éditeur(s)}

Revue Santé mentale au Québec

\section{ISSN}

0383-6320 (imprimé)

1708-3923 (numérique)

\section{Découvrir la revue}

\section{Citer cet article}

Perreault, M., Mechakra-Tahiri, D.-S., Fleury, M.-J., Touré, E., Mitchell, E. \& Caron, J. (2013). Facteurs associés à l'exposition élevée aux médicaments psychotropes identifiés lors d'une étude épidémiologique montréalaise. Santé mentale au Québec, 38(1), 259-278. https://doi.org/10.7202/1019196ar

\section{Résumé de l'article}

L'article examine les associations entre l'exposition élevée aux médicaments psychotropes, la présence d'un diagnostic de trouble mental et les caractéristiques sociodémographiques chez la population adulte. Un questionnaire a été passé par entrevue auprès de 2433 personnes de 15 ans et plus dans le cadre de l'enquête ZEPSOM (zone épidémiologique du sud-ouest de Montréal). Les déterminants de leur consommation de psychotropes ont fait l'objet d'analyses bivariées et de régression logistique multivariée. Une association bivariée significative a été observée entre la consommation de somnifères, d'anxiolytiques et d'antidépresseurs et être plus âgé, être une femme, vivre seul, avoir un niveau d'instruction et de revenu faible, de ne pas avoir occupé un emploi au cours des 12 mois précédant l'enquête et présenter un trouble mental. Une exposition élevée aux différents médicaments psychotropes (trois ou plus) a été rapportée chez 3,1 \% des répondants. Toutes choses étant égales, elle augmente avec l'âge, le fait de vivre seul, ne pas avoir occupé d'emploi au cours de la dernière année et de présenter un trouble mental. Les résultats suggèrent la nécessité de considérer l'isolement social et la prévalence des troubles mentaux pour contextualiser l'exposition élevée aux médicaments psychotropes puisque la polypharmacie peut présenter des risques importants si elle ne s'inscrit pas dans la logique d'un protocole d'intervention clinique cohérent.
Tous droits réservés (C Département de psychiatrie de l’Université de Montréal, 2013 e document est protégé par la loi sur le droit d'auteur. L'utilisation des services d'Érudit (y compris la reproduction) est assujettie à sa politique d'utilisation que vous pouvez consulter en ligne.

https://apropos.erudit.org/fr/usagers/politique-dutilisation/ 


\title{
Facteurs associés à l'exposition élevée aux médicaments psychotropes identifiés lors d'une étude épidémiologique montréalaise
}

\author{
Michel Perreault, Ph.D. ${ }^{a, b}$ \\ Mechakra-Tahiri Djemaâ-Samia, Ph.D. ${ }^{a}$ \\ Marie-Josée Fleury, Ph.D. ${ }^{a, b}$ \\ El Hadj Touré, Ph.D. ${ }^{\text {a }}$ \\ Emma Mitchella \\ Jean Caron, Ph.D. ${ }^{\mathrm{a}, \mathrm{b}}$
}

\begin{abstract}
RÉSUMÉ L'article examine les associations entre l'exposition élevée aux médicaments psychotropes, la présence d'un diagnostic de trouble mental et les caractéristiques sociodémographiques chez la population adulte. Un questionnaire a été passé par entrevue auprès de 2433 personnes de 15 ans et plus dans le cadre de l'enquête ZEPSOM (zone épidémiologique du sud-ouest de Montréal). Les déterminants de leur consommation de psychotropes ont fait l'objet d'analyses bivariées et de régression logistique multivariée. Une association bivariée significative a été observée entre la consommation de somnifères, d'anxiolytiques et d'antidépresseurs et être plus âgé, être une femme, vivre seul, avoir un niveau d'instruction et de revenu faible, de ne pas avoir occupé un emploi au cours des 12 mois précédant l'enquête et présenter un trouble mental. Une exposition élevée aux différents médicaments psychotropes (trois ou plus) a été rapportée chez 3,1\% des répondants. Toutes choses étant égales, elle augmente avec l'âge, le fait de vivre seul, ne pas avoir occupé d'emploi au cours de la dernière année et de présenter un trouble mental. Les résultats suggèrent la nécessité de considérer l'isolement social et la prévalence des troubles mentaux pour contextualiser l'exposition élevée aux médicaments psychotropes puisque la polypharmacie peut présenter des risques importants si elle ne s'inscrit pas dans la logique d'un protocole d'intervention clinique cohérent.
\end{abstract}

a. Institut universitaire en santé mentale Douglas, Montréal, Québec.

b. Département de psychiatrie, Université McGill, Montréal, Québec. 
MOTS CLÉS médicaments psychotropes, exposition élevée, déterminants, trouble mental.

\section{Factors associated with high exposure to psychotropic drugs in a Montreal catchment epidemiological area}

ABSTRACT This article examine the associations between elevated exposure to psychotropic medications, presence of a diagnosis of mental illness, and sociodemographic characteristics in the adult population. A questionnaire was administered by interview to 2433 individuals aged 15 and over in the epidemiological catchment area of South-West Montreal. The determinants of psychotropic medication consumption were analyzed using bivariate analysis and multivariate logistic regression. A significant association was observed between the consumption of sleeping medications, anxiolytics, and antidepressants and being older, female, living alone, having a low level of education and income, being unemployed during the 12 months preceding the study, and presence of a mental disorder. An elevated exposure to different psychotropic medications (three or more) was reported in $3.1 \%$ of the respondants. All things being equal, this increased with age, living alone, being unemployed over the course of the last year, and presence of a mental illness. The results suggest that it is necessary to consider social isolation and prevalence of mental illness in order to contextualize the elevated exposure to psychotropic medication. Polypharmacy may indeed pose important risks if it does not follow the logic of a coherent clinical intervention.

KEYWORDS psychotropic medication, elevated exposure, determinants, mental illness

\section{Introduction}

La surconsommation de médicaments psychotropes constitue un problème de santé publique majeur par son ampleur et par l'importance du coût social et économique s'y rattachant (Alessi-Severini et al., 2008; Beck et al., 2005; Hemels et al., 2002). Ce problème est d'autant plus préoccupant que l'on rapporte une augmentation croissante de l'utilisation de ces médicaments dans la plupart des pays du monde (Alla \& Baumann, 2003; Chien et al., 2008; Empereur et al., 2003; Hall et al., 2003; Reseland et al., 2006). D’ailleurs, au Québec, les psychotropes sont les médicaments les plus prescrits chez les adhérents à la Régie de l'assurance maladie du Québec (RAMQ) avec 11,2\% d'ordonnances en 1998 et en 2001 (Comité permanent de lutte à la 
toxicomanie, 2003). L'association entre certains déterminants sociodémographiques et la consommation de psychotropes a été rapportée dans plusieurs études (Cohidon et al., 2005; Enquête sur la santé dans les collectivités canadiennes (cycle 1.2), 2009; Hollingworth et al., 2003; Percudani et al., 2005). Ainsi, les personnes de sexe féminin, âgées, vivant seules, ayant un faible revenu sont plus susceptibles de consommer des médicaments psychotropes. Toutefois, il importe d'examiner l'influence que pourrait exercer aussi la présence de troubles mentaux sur ce type de consommation.

La recherche systématique sur l'usage de multiples médicaments psychotropes est limitée (Patrick et al., 2005). Certes, des données existent sur la prescription de plus d'un médicament psychotrope (c.-à-d. antidépresseurs et psychostimulants ou antidépresseurs et anxiolytiques), mais il importe d'étudier en profondeur cette pratique passablement répandue. La polyconsommation peut en effet être à l'origine d'un problème de santé publique majeur en raison de son association avec des réactions indésirables des médicaments. Elle peut même, dans les cas critiques, mener jusqu'au décès (Klarin et al., 2005; Lau et al., 2005; Lesen et al., 2010 ; Perri et al., 2005). Bien que la plupart des guides de pratique recommandent principalement la monothérapie, il importe donc d'évaluer le recours à plus d'un médicament psychotrope (Preskorn \& Lacey, 2007), tout spécialement lorsqu'il atteint un taux élevé d'exposition, qui accroît non seulement le coût de la thérapie mais aussi les risques d'effets adverses.

La consommation élevée de médicaments psychotropes est généralement considérée préoccupante lorsque trois types de médicaments psychotropes ou plus sont pris par des jeunes ou des personnes âgées ou encore que quatre types de ces médicaments ou plus sont consommés par des adultes (Essock et al., 2009; Johnell et al., 2007; Lesen et al., 2010).

La présente étude a été menée auprès de la population du sud-ouest de Montréal dans le cadre d'une étude épidémiologique de zone circonscrite. La consommation de trois classes de médicaments psychotropes ou plus a été retenue comme étant une mesure conservatrice d'exposition élevée. L'objectif de l'étude est d'identifier les facteurs sociodémographiques et diagnostics de troubles de santé mentale associés à l'utilisation de médicaments psychotropes et, plus spécifiquement, à ceux reliés à une exposition élevée à ces substances au sein de la population adulte. 


\section{Méthode}

\section{Échantillonnage et collecte des données}

La population cible est constituée de personnes âgées de 15 ans et plus du sud-ouest de Montréal recrutées dans le cadre de l'enquête ZEPSOM (Caron et al., 2012). L'échantillon se veut représentatif de cette population et est stratifié selon la location géographique, la densité de la population et le statut socioéconomique des territoires. Le nombre de personnes âgées de 15 à 65 ans était de 198585 alors que la population totale de la zone épidémiologique comptait 269720 habitants.

Pour le recrutement, 3408 adresses ont été aléatoirement sélectionnées dans une liste d'adresses du rôle d'évaluation des propriétés de la ville de Montréal. Une seule personne par ménage a été choisie en utilisant les procédures et les critères de l'Enquête nationale sur la santé (Enquête nationale sur la santé de la population (ENSP), 20032005). Parmi les 4269 candidats répondant aux critères, 1405 ont refusé de participer à l'étude (32,9\%), 730 répondants (17\%) ont accepté d'y participer lors du premier contact, mais se sont désistés par la suite, pour l'entrevue. Au total, l'échantillon final regroupe 2433 participants dont 600 dans chaque arrondissement: Saint-Henri/Pointe St-Charles (612), Lachine/Dorval (603), LaSalle (584) et Verdun (635). Le taux de réponses est de $48,7 \%$.

Le questionnaire a été passé en 2006 lors d'une entrevue personnelle à domicile ou dans un bureau spécialement aménagé. Les participants ont signé deux formulaires: l'un pour accorder leur consentement à participer à l'étude et un autre autorisant l'équipe à les contacter si le questionnaire permettait de détecter un trouble psychiatrique chez eux alors qu'ils ne recevaient pas de services de santé mentale. Le projet a été approuvé par le comité d'éthique de la recherche de l'Institut universitaire en santé mentale Douglas.

\section{Variables et mesures}

Les troubles mentaux considérés sont les troubles anxieux (trouble panique, phobie sociale et agoraphobie), les troubles de l'humeur (dépression majeure et manie) et la toxicomanie (dépendance à l'alcool et/ou aux drogues illicites). Ils ont été évalués à l'aide du «Composite International Diagnostic Interview: CIDI» (Kessler et al., 1998).

La consommation de médicaments psychotropes (somnifères, régulateurs de l'humeur, anxiolytiques, antidépresseurs, antipsychotiques et stimulants) au cours de la dernière année a été évaluée à l'aide des 
questions suivantes, tirées de l'Enquête sur la santé dans les collectivités canadiennes (Enquête sur la santé dans les collectivités canadiennes (cycle 1.2), 2009): Au cours des 12 derniers mois, avez-vous pris : 1) des médicaments pour vous aider à dormir (tels que Imovane, Nytol ou Starnoc); 2) des médicaments pour réduire l'anxiété ou la «nervosité» (tels que Ativan, Valium ou Serax); 3) des stabilisateurs de l'humeur (tels que Lithium, Tegretol ou Epival); 4) des antidépresseurs (tels que Prozac, Paxil ou Effexor); 5) des médicaments pour le traitement de comportements psychotiques (tels que Haldol, Risperdal ou Seroquel); et 6) des stimulants (tels que Ritalin, Dexedrine ou Alertec).

Le nombre de médicaments psychotropes consommés renvoie au nombre de réponses aux six questions concernant les classes de médicaments. Ainsi l'exposition élevée aux médicaments psychotropes a été mesurée en regroupant les participants conformément à la procédure de Lesen (2010), c'est-à-dire selon qu'ils font usage ou non d'au moins trois psychotropes. Le sexe, l'âge, l'état matrimonial, le niveau d'instruction, le statut d'emploi au cours des 12 derniers mois et le revenu du ménage constituent les variables sociodémographiques retenues.

\section{Analyses statistiques}

Avant de faire l'objet d'analyses statistiques à l'aide du logiciel SAS, les données ont été pondérées en fonction des proportions de la structure du groupe d'âge, du sexe et de l'arrondissement dans la population $(n=2433)$. Ainsi, les différences dans le profil de consommation de classes de médicaments psychotropes selon les caractéristiques sociodémographiques des participants et des diagnostics ont été examinées à l'aide du test de Chi-2. Les diagnostics de troubles de l'humeur, de troubles anxieux et de toxicomanie ont fait l'objet d'analyses. Les variables associées à la consommation de trois médicaments psychotropes ou plus dans les analyses bivariées $(\mathrm{Chi}-2: \mathrm{p}<0,05)$ ont été retenues pour l'analyse multivariée. Le choix du diagnostic à inclure dans le modèle multivarié s'est fait par la construction de modèles de régression logistique pour chacun des diagnostics de troubles mentaux en contrôlant pour les variables sociodémographiques. La comparaison de la C-statistique obtenue pour chacun des modèles indique que le modèle fondé sur la présence d'au moins un trouble mental au cours des 12 derniers mois s'avère le plus adéquat. Un modèle de régression logistique final a donc été testé afin d'examiner les associations entre les variables retenues et la consommation d'au moins trois psychotropes. 


\section{Résultats}

Le tableau 1 présente les caractéristiques sociodémographiques des participants ainsi que la présence d'un diagnostic de trouble mental. La moyenne d'âge des participants est de $40,7 \pm 14,1$ ans ; $49 \%$ sont des hommes; $29,4 \%$ sont mariés ; $27 \%$ ont un revenu inférieur à 25000 \$ et la majorité d'entre eux $(71,9 \%)$ ont une scolarité de niveau secondaire. La prévalence des troubles mentaux au cours de la dernière année pour la population de 15 ans et plus est respectivement de 2,6\% pour les troubles anxieux seulement (trouble panique, phobie sociale et agoraphobie), 5,9\% pour les troubles de l'humeur seuls (dépression majeure et/ou manie) et $6,3 \%$ pour la «toxicomanie» (dépendance à l'alcool: 4,1\%; dépendance aux drogues illicites: $3,2 \%$ ).

Comme l'indique le tableau 2, les somnifères sont les plus utilisés $(10,3 \%)$, suivis des antidépresseurs $(9,1 \%)$ et des anxiolytiques $(7,8 \%)$. Pour ces trois classes de médicaments, la consommation est significativement plus élevée chez les femmes, les personnes moins scolarisées et celles ayant un revenu faible. Les personnes plus âgées ainsi que les personnes vivant seules et celles qui ont été sans emploi au cours de la dernière année sont également plus nombreuses à consommer ces médicaments. Concernant les régulateurs de l'humeur et les antipsychotiques, leur consommation est associée au statut matrimonial, à un faible revenu et à l'absence d'emploi chez les répondants. En ce qui a trait aux stimulants, aucune différence significative n'a été observée en fonction des caractéristiques sociodémographiques des participants.

Contrairement aux troubles anxieux et à la toxicomanie, les troubles de l'humeur sont associés à la consommation d'un plus grand nombre de médicaments psychotropes (tableau 3). Parmi les personnes présentant un diagnostic de trouble anxieux, $13,5 \%$ ont pris un anxiolytique au cours de la dernière année, alors que $17,2 \%$ ont pris un antidépresseur. Plus du tiers des personnes souffrant d'un trouble de l'humeur ont consommé un antidépresseur (35,1\%), alors que 27,1\% d'entre elles ont pris un somnifère et $21,4 \%$ un anxiolytique.

Aussi, le tableau 4 montre que $3,1 \%$ des participants affirment avoir consommé trois psychotropes ou plus au cours des douze derniers mois. À l'exception du niveau de scolarité et du diagnostic des troubles anxieux seul, toutes les variables sociodémographiques et de diagnostics prises isolément sont significativement associées à l'exposition élevée aux médicaments psychotropes. 
TABLEAU 1 Distribution des caractéristiques sociodémographiques et de la prévalence des troubles mentaux (diagnostics exclusifs*) sur 12 mois $(n=2433)$

\begin{tabular}{|c|c|}
\hline Caractéristiques & $\%$ \\
\hline \multicolumn{2}{|l|}{ Profil sociodémographique } \\
\hline \multicolumn{2}{|l|}{ Sexe } \\
\hline Femme & 51,4 \\
\hline Homme & 48,6 \\
\hline \multicolumn{2}{|l|}{ Âge } \\
\hline $15-24$ & 16,1 \\
\hline $25-34$ & 20,7 \\
\hline $35-44$ & 20,8 \\
\hline $45-54$ & 20,9 \\
\hline $55+$ & 21,5 \\
\hline \multicolumn{2}{|l|}{ État matrimonial } \\
\hline Célibataire & 38,0 \\
\hline Marié & 29,4 \\
\hline Séparé & 2,8 \\
\hline Union libre & 15,9 \\
\hline Divorcé & 12,4 \\
\hline Veuf & 1,6 \\
\hline \multicolumn{2}{|l|}{ Niveau d'instruction } \\
\hline Moins que le secondaire & 16,0 \\
\hline Secondaire & 12,1 \\
\hline Postsecondaire & 71,9 \\
\hline En emploi durant les 12 derniers mois & 78,6 \\
\hline \multicolumn{2}{|l|}{ Revenu du ménage } \\
\hline $0-25000$ & 27,0 \\
\hline $25000-50000$ & 28,1 \\
\hline $50000-75000$ & 18,5 \\
\hline $75000+$ & 26,4 \\
\hline \multicolumn{2}{|l|}{ Diagnostics exclusifs } \\
\hline Troubles anxieux seulement ${ }^{1}$ & 2,6 \\
\hline Troubles de l'humeur seulement ${ }^{2}$ & 5,9 \\
\hline Toxicomanie $^{3}$ & 6,3 \\
\hline Troubles anxieux et troubles de l'humeur ${ }^{4}$ & 2,5 \\
\hline Troubles anxieux et/ou troubles de l'humeur et toxicomanie ${ }^{5}$ & 2,2 \\
\hline Aucun trouble mental ${ }^{6}$ & 81,1 \\
\hline
\end{tabular}

\footnotetext{
* Une personne ne peut se retrouver que dans l'une des six catégories de diagnostic présentées dans le tableau.

1. Trouble panique, et/ou phobie sociale, et/ou agoraphobie; mais n'ayant aucun des troubles suivants: dépression majeure, manie, dépendance à l'alcool, dépendance aux médicaments

2. Dépression majeure et/ou manie; mais n'ayant aucun des troubles suivants: trouble panique, phobie sociale, agoraphobie, dépendance à l'alcool, consommation de drogues

3. Dépendance à l'alcool et/ou drogues illicites

4. Au moins un des troubles anxieux et au moins un des troubles de l'humeur

5. Au moins un des troubles anxieux et/ou au moins un des troubles de l'humeur et toxicomanie

6. N'ayant aucun des troubles suivants: dépression majeure, manie, trouble panique, phobie sociale, agoraphobie, dépendance à l'alcool, dépendance à la drogue
} 
TABLEAU 2 Consommation de médicaments psychotropes (prescrits ou non), au cours d'une période de 12 mois, selon les caractéristiques sociodémographiques des participants et la présence d'un diagnostic de trouble mental $(n=2433)$

\begin{tabular}{|c|c|c|c|c|c|c|}
\hline Caractéristiques & Somnifères & Anxiolytiques & $\begin{array}{l}\text { Régulateurs de } \\
\text { l'humeur }\end{array}$ & Antidépresseurs & Antipsychotiques & Stimulants \\
\hline \multicolumn{7}{|c|}{$\%($ (IC95 \%) } \\
\hline Population totale & $10,3(9,0-11,6)$ & $7,8(6,6-8,9)$ & $2,4(1,7-3,1)$ & $9,1(8,0-10,3)$ & $2,3(1,7-3,0)$ & $0,6(0,2-0,9)$ \\
\hline \multicolumn{7}{|l|}{ Démographiques } \\
\hline $\begin{array}{l}\text { Sexe } \\
\text { Hommes }\end{array}$ & $\begin{array}{l}p<0,05 \\
7,9(6,1-9,7)\end{array}$ & $\begin{array}{l}p<0,05 \\
5,4(3,8-6,9)\end{array}$ & $\begin{array}{l}\text { n.s. } \\
2,7(1,6-3,8)\end{array}$ & $\begin{array}{l}p<0,05 \\
5,7(4,2-7,2)\end{array}$ & $\begin{array}{l}\text { n.s. } \\
2,8(1,7-3,9)\end{array}$ & $\begin{array}{l}\text { n.s. } \\
0,6(0,1-1,1)\end{array}$ \\
\hline Femmes & $12,6(10,8-14,4)$ & $10,0(8,4-11,6)$ & $2,2(1,4-2,9)$ & $12,3(10,6-14,1)$ & $1,9(1,1-2,6)$ & $0,5(0,1-0,9)$ \\
\hline $\begin{array}{l}\text { Âge } \\
15-24 \text { ans }\end{array}$ & $\begin{array}{l}\mathrm{p}<0,05 \\
2,2(0,5-3,9)\end{array}$ & $\begin{array}{l}p<0,05 \\
2,2(0,3-4,1)\end{array}$ & $\begin{array}{l}p<0,05 \\
0,6(0,0-1,4)\end{array}$ & $\begin{array}{l}p<0,05 \\
1,2(0,0-2,4)\end{array}$ & $\begin{array}{l}\text { n.s. } \\
1,2(0,0-2,6)\end{array}$ & $\begin{array}{l}\text { n.s. } \\
0,8(0,0-1,8)\end{array}$ \\
\hline $25-44$ ans & $9,4(7,5-11,2)$ & $6,4(4,9-8,0)$ & $2,5(1,6-3,5)$ & $8,9(7,1-10,6)$ & $2,0(1,1-2,9)$ & $0,4(0,0-0,8)$ \\
\hline 45 ans et plus & $14,3(12,1-16,6)$ & $11,2(9,2-13,2)$ & $2,9(1,8-4,1)$ & $12,4(10,3-14,5)$ & $3,0(1,8-4,2)$ & $0,6(0,1-1,1)$ \\
\hline $\begin{array}{l}\text { État matrimonial } \\
\text { Célibataire }\end{array}$ & $\begin{array}{l}p<0,05 \\
10,2(8,2-12,3)\end{array}$ & $\begin{array}{l}p<0,05 \\
8,2(6,3-10,0)\end{array}$ & $\begin{array}{l}\mathrm{p}<0,05 \\
3,1(1,9-4,3)\end{array}$ & $\begin{array}{l}p<0,05 \\
8,3(6,5-10,2)\end{array}$ & $\begin{array}{l}p<0,05 \\
3,6(2,3-4,8)\end{array}$ & $\begin{array}{l}\text { n.s. } \\
1,0(0,3-1,7)\end{array}$ \\
\hline Avec un partenaire & $7,3(5,7-8,9)$ & $4,7(3,4-6,0)$ & $1,2(0,5-1,8)$ & $7,0(5,4-8,4)$ & $0,9(0,2-1,5)$ & $0,2(0,0-0,5)$ \\
\hline Séparé/veuf/divorcé & $18,3(14,3-22,3)$ & $15,3(11,6-18,9)$ & $4,2(1,9-6,6)$ & $16,8(13,1-20,6)$ & $3,6(1,5-5,7)$ & $0,5(0,0-1,1)$ \\
\hline \multicolumn{7}{|l|}{ Socioéconomiques } \\
\hline $\begin{array}{l}\text { Niveau d'instruction } \\
\text { < Secondaire }\end{array}$ & $\begin{array}{l}p<0,05 \\
16,7(9,7-23,7)\end{array}$ & $\begin{array}{l}p<0,05 \\
16,3(9,4-23,3)\end{array}$ & $\begin{array}{l}\text { n.s. } \\
0,8(0,0-1,8)\end{array}$ & $\begin{array}{l}p<0,05 \\
10,9(5,6-16,1)\end{array}$ & $\begin{array}{l}\text { n.s. } \\
3,9(0,3-7,5)\end{array}$ & $\begin{array}{l}\text { n.s. } \\
1,7(0,0-4,3)\end{array}$ \\
\hline Secondaire & $11,4(7,5-15,2)$ & $10,0(6,5-13,5)$ & $2,0(0,4-3,6)$ & $8,9(5,6-12,2)$ & $2,0(0,4-3,6)$ & $0,6(0,0-1,6)$ \\
\hline Postsecondaire & $9,8(8,4-11,1)$ & $6,9(5,7-8,1)$ & $2,6(1,8-3,3)$ & $9,1(7,8-10,3)$ & $2,3(1,5-3,0)$ & $0,5(0,2-0,8)$ \\
\hline $\begin{array}{l}\text { Revenu du ménage } \\
0-25000\end{array}$ & $\begin{array}{l}p<0,05 \\
12,8(10,0-15,6)\end{array}$ & $\begin{array}{l}p<0,05 \\
11,1(8,5-13,7)\end{array}$ & $\begin{array}{l}p<0,05 \\
3,9(2,2-5,6)\end{array}$ & $\begin{array}{l}p<0,05 \\
12,5(9,8-15,2)\end{array}$ & $\begin{array}{l}p<0,05 \\
4,0(2,3-5,6)\end{array}$ & $\begin{array}{l}\text { n.s. } \\
0,7(0,01-1,4)\end{array}$ \\
\hline $25000-50000$ & $10,9(8,3-13,5)$ & $8,2(5,8-10,6)$ & $2,7(1,3-4,1)$ & $9,9(7,5-12,3)$ & $3,2(1,7-4,7)$ & $0,5(0,0-1,1)$ \\
\hline $50000-75000$ & $11,2(7,9-14,4)$ & $8,0(5,2-10,8)$ & $2,8(1,1-4,6)$ & $9,1(6,2-12,0)$ & $1,5(0,0-3,0)$ & $0,6(0,0-1,5)$ \\
\hline $75000+$ & $7,6(5,3-9,9)$ & $4,5(2,7-6,2)$ & $0,9(0,1-1,8)$ & $7,1(5,0-9,3)$ & $1,2(0,09-2,4)$ & $0,5(0,0-1,2)$ \\
\hline \multicolumn{7}{|l|}{ En emploi, durant les } \\
\hline $\begin{array}{l}12 \text { derniers mois } \\
\text { Oui }\end{array}$ & $\begin{array}{l}p<0,05 \\
9,2(7,8-10,6)\end{array}$ & $\begin{array}{l}p<0,05 \\
5,9(4,7-7,0)\end{array}$ & $\begin{array}{l}p<0,05 \\
1,6(1,0-2,3)\end{array}$ & $\begin{array}{l}\mathrm{p}<0,05 \\
7,3(6,1-8,5)\end{array}$ & $\begin{array}{l}p<0,05 \\
1,6(1,0-2,3)\end{array}$ & $\begin{array}{l}\text { n.s. } \\
0,6(0,2-1,0)\end{array}$ \\
\hline Non & $14,6(11,4-17,7)$ & $14,6(11,5-17,7)$ & $5,3(3,3-7,3)$ & $15,8(12,6-19,0)$ & $4,9(2,9-6,9)$ & $0,4(0,0-0,9)$ \\
\hline
\end{tabular}




\section{TABLEAU 3 Consommation de médicaments psychotropes (prescrits ou non) au cours d'une période de 12 mois, selon la présence d'un diagnostic d'un trouble mental}

\begin{tabular}{|c|c|c|c|c|c|c|}
\hline $\begin{array}{l}\text { Présence d'un } \\
\text { diagnostic }\end{array}$ & Somnifères & Anxiolytiques & $\begin{array}{l}\text { Régulateurs de } \\
\text { I'humeur }\end{array}$ & Antidépresseurs & Antipsychotiques & Stimulants \\
\hline \multicolumn{7}{|c|}{$\%$ (IC95 \%) } \\
\hline $\begin{array}{l}\text { Troubles anxieux } \\
\text { seulement }\end{array}$ & n.s. & n.s. & n.s. & $p<0,05$ & n.s. & n.s. \\
\hline Oui & $11,1(3,6-18,7)$ & $13,5(5,7-21,4)$ & $3,6(0,0-7,7)$ & $17,2(8,6-25,7)$ & $1,6(0,0-4,7)$ & $1,6(0,0-4,8)$ \\
\hline Non & $10,3(9,0-11,6)$ & $7,6(6,5-8,7)$ & $2,4(1,7-3,0)$ & $8,9(7,8-10,1)$ & $2,3(1,7-3,0)$ & $0,5(0,2-0,8)$ \\
\hline \multicolumn{7}{|l|}{ Troubles de I'humeur } \\
\hline seulement & n.s. & $p<0,05$ & $p<0,05$ & $p<0,05$ & $p<0,05$ & $p<0,05$ \\
\hline Oui & $27,1(20,0-34,2)$ & $21,4(14,9-28,0)$ & $8,1(3,6-12,6)$ & $35,1(27,5-42,6)$ & $8,0(3,4-12,5)$ & $2,0(0,0-4,3)$ \\
\hline Non & $9,0(7,8-10,3)$ & $6,7(5,6-7,8)$ & $2,0(1,3-2,6)$ & $7,2(6,1-8,2)$ & $1,9(1,3-2,5)$ & $0,4(0,2-0,7)$ \\
\hline Toxicomanie & n.s. & n.s. & n.s. & $p<0,05$ & $p<0,05$ & n.s. \\
\hline Oui & $15,1(9,0-21,1)$ & $10,0(5,2-14,7)$ & $3,9(0,6-7,3)$ & $16,4(10,2-22,6)$ & $6,1(2,0-10,3)$ & $1,3(0,0-3,2)$ \\
\hline Non & $9,9(8,6-11,2)$ & $7,5(6,3-8,6)$ & $2,3(1,7-3,0)$ & $8,7(7,5-9,9)$ & $2,1(1,4-2,7)$ & $0,5(0,2-0,8)$ \\
\hline $\begin{array}{l}\text { Troubles anxieux et } \\
\text { troubles de I'humeur }\end{array}$ & $p<0,05$ & $\mathrm{p}<0,05$ & $p<0,05$ & $p<0,05$ & $p<0,05$ & $p<0,05$ \\
\hline Oui & $35,6(22,8-48,5)$ & $35,7(22,7-48,6)$ & $10,6(2,5-18,7)$ & $44,2(30,5-57,8)$ & $12,0(2,9-21,0)$ & $3,8(0,0-9,2)$ \\
\hline Non & $9,7(8,5-11,0)$ & $7,1(6,0-8,2)$ & $2,2(1,6-2,9)$ & $8,3(7,2-9,5)$ & $2,1(1,5-2,8)$ & $0,5(0,2-0,8)$ \\
\hline $\begin{array}{l}\text { Troubles anxieux et/ou } \\
\text { troubles de I'humeur } \\
\text { et toxicomanie }\end{array}$ & n.s. & n.s. & n.s. & $p<0,05$ & $p<0,05$ & n.s. \\
\hline Oui & $18,4(7,2-29,5)$ & $17,2(6,5-27,8)$ & $6,8(0,0-14,4)$ & $26,3(13,5-39,2)$ & $11,1(1,5-20,8)$ & $2,7(0,0-7,9)$ \\
\hline Non & $10,1(8,9-11,4)$ & $7,6(6,4-8,7)$ & $2,3(1,6-3,0)$ & $8,7(7,6-9,9)$ & $2,1(1,5-2,8)$ & $0,5(0,2-0,8)$ \\
\hline $\begin{array}{l}\text { N'importe } \\
\text { quel trouble }\end{array}$ & $p<0,05$ & n.s. & $p<0,05$ & $p<0,05$ & $p<0,05$ & n.s. \\
\hline Oui & $21,9(17,6-26,2)$ & $18,2(14,3-22,1)$ & $6,2(3,7-8,7)$ & $27(22,4-31,5)$ & $6,2(3,6-8,8)$ & $1,8(0,4-3,2)$ \\
\hline Non & $8,1(6,8-9,3)$ & $5,7(4,7-6,8)$ & $1,7(1,0-2,3)$ & $5,6(4,6-6,7)$ & $1,6(1,0-2,2)$ & $0,3(0,1-0,6)$ \\
\hline
\end{tabular}


TABLEAU 4 Profil de consommation des psychotropes (prescrits ou non) au cours d'une période de 12 mois, selon les caractéristiques sociodémographiques et la présence d'un diagnostic de trouble mental

\begin{tabular}{|c|c|c|}
\hline Caractéristiques & $\begin{array}{l}\text { Consommation de trois } \\
\text { psychotropes ou plus }\end{array}$ & $p\left(x^{2}\right)$ \\
\hline & $\%($ IC95 \%) & \\
\hline Population totale & $\mathbf{3 , 1}(2,4-3,8)$ & \\
\hline \multicolumn{3}{|l|}{ Démographiques } \\
\hline $\begin{array}{l}\text { Sexe } \\
\text { Hommes } \\
\text { Femmes }\end{array}$ & $\begin{array}{l}2,3(1,3-3,3) \\
3,9(2,8-4,9)\end{array}$ & 0,05 \\
\hline $\begin{array}{l}\text { Âge } \\
15-24 \text { ans } \\
25-44 \text { ans } \\
45 \text { ans et plus }\end{array}$ & $\begin{array}{l}1,1(0,0-2,3) \\
3,0(2,0-4,0) \\
4,0(2,7-5,3)\end{array}$ & 0,04 \\
\hline $\begin{array}{l}\text { État matrimonial } \\
\text { Célibataire } \\
\text { Vivant avec un partenaire } \\
\text { Séparé/veuf/divorcé }\end{array}$ & $\begin{array}{l}3,8(2,5-5,0) \\
1,4(0,7-2,1) \\
6,2(3,6-8,9)\end{array}$ & $<0,0001$ \\
\hline \multicolumn{3}{|l|}{ Socioéconomiques } \\
\hline $\begin{array}{l}\text { Niveau d'instruction } \\
\text { Moins que le secondaire } \\
\text { Secondaire } \\
\text { Postsecondaire }\end{array}$ & $\begin{array}{l}6,4(2,1-10,7) \\
3,4(1,2-5,5) \\
2,9(2,1-3,6)\end{array}$ & 0,09 \\
\hline $\begin{array}{l}\text { Revenu du ménage } \\
0-25000 \\
25000-50000 \\
50000-75000 \\
75000+\end{array}$ & $\begin{array}{l}5,3(3,4-7,1) \\
3,3(1,8-4,8) \\
3,4(1,3-5,4) \\
1,3(0,3-2,3)\end{array}$ & $<0,01$ \\
\hline $\begin{array}{l}\text { En emploi, durant les } 12 \text { derniers mois } \\
\text { Oui } \\
\text { Non }\end{array}$ & $\begin{array}{l}2,0(1,3-2,7) \\
7,2(5,0-9,5)\end{array}$ & $<0,0001$ \\
\hline \multicolumn{3}{|l|}{ Présence d'un diagnostic } \\
\hline $\begin{array}{l}\text { Troubles anxieux seulement } \\
\text { Oui } \\
\text { Non }\end{array}$ & $\begin{array}{l}3,9(0,0-8,3) \\
3,1(2,3-3,8)\end{array}$ & 0,7 \\
\hline $\begin{array}{l}\text { Troubles de l'humeur seulement } \\
\text { Oui } \\
\text { Non }\end{array}$ & $\begin{array}{l}13,2(7,7-18,7) \\
2,3(1,7-3,0)\end{array}$ & $<0,0001$ \\
\hline $\begin{array}{l}\text { Toxicomanie } \\
\text { Oui } \\
\text { Non }\end{array}$ & $\begin{array}{l}6,6(2,5-10,7) \\
3,0(2,2-3,6)\end{array}$ & 0,01 \\
\hline $\begin{array}{l}\text { Troubles anxieux et troubles de l'humeur } \\
\text { Oui } \\
\text { Non }\end{array}$ & $\begin{array}{l}26,4(14,8-38,0) \\
2,6(1,9-3,3)\end{array}$ & $<0,0001$ \\
\hline $\begin{array}{l}\text { Troubles anxieux et/ou troubles } \\
\text { de l'humeur et toxicomanie }\end{array}$ & & $<0,0001$ \\
\hline $\begin{array}{l}\text { Oui } \\
\text { Non }\end{array}$ & $\begin{array}{l}13,6(3,5-23,6) \\
2,9(2,2-3,6)\end{array}$ & \\
\hline $\begin{array}{l}\text { N'importe quel trouble } \\
\text { Oui } \\
\text { Non }\end{array}$ & $\begin{array}{l}10,7(7,6-13,9) \\
1,6(1,0-2,2)\end{array}$ & $<0,0001$ \\
\hline
\end{tabular}


TABLEAU 5 Modèle de régression logistique multivariée sur la consommation de trois classes de médicaments psychotropes ou plus

\begin{tabular}{lll}
\hline Prédicteurs & Rapport de cote ajusté & \multicolumn{1}{c}{ IC95\% } \\
\hline Sexe & 1 & - \\
$\quad$ Femme & $0,75^{\text {n.s }}$ & $0,45-1,26$ \\
Homme & & \\
Âge & 1 & - \\
$15-24$ & $2,98^{\text {n.s }}$ & $1,00-9,00$ \\
$25-44$ & $3,16^{*}$ & $1,04-9,64$ \\
$45+$ & & - \\
État matrimonial & 1 & $1,38-5,61$ \\
Vivant avec un partenaire & $2,80^{* *}$ & $1,78-7,90$ \\
Célibataire & $3,80^{* * *}$ & - \\
Séparé/veuf/divorcé & & $0,68-2,51$ \\
Revenu du ménage & 1 & $0,93-4,29$ \\
$\quad \leq 25000$ & $1,31^{\text {n.s }}$ & $0,37-2,40$ \\
$25000-50$ 000 & $2,00^{\text {n.s }}$ & \\
$50000-75$ 000 & $0,93^{\text {n.s }}$ & - \\
$\geq 75$ 000+ & & $0,17-0,51$ \\
En emploi, durant les 12 derniers mois & 1 & - \\
Non & $0,29^{* * *}$ & $3,70-10,30$ \\
Oui & & \\
N'importe quel trouble de santé mentale & 1 & $6,12^{* * *}$ \\
Non & & \\
Oui & & \\
\hline
\end{tabular}

${ }^{*} \mathrm{p}<0,05 ;{ }^{* *} \mathrm{p}<0,01 ;{ }^{* * *} \mathrm{p}<0,001$

ns: non significatif

Les résultats de l'analyse multivariée (tableau 5) indiquent que la probabilité d'utilisation de trois types de médicaments psychotropes ou plus est plus élevée chez les personnes âgées de 45 ans et plus $(\mathrm{RC}=$ 3,16; IC95\%: 1,04-9,64) que chez les jeunes de 15 à 24 ans, chez les personnes séparées, divorcées ou veuves $(\mathrm{RC}=3,80 ; \mathrm{IC} 95 \%: 1,78-7,90)$ ou célibataires $(\mathrm{RC}=2,80$; IC95\%:1,38-5,61) que chez les personnes vivant avec un partenaire et chez celles qui n'ont pas occupé un emploi au cours des 12 derniers mois ( $R C=0,29$; IC95\%: 0,17-0,51). Le sexe et le revenu demeurent, toutes choses étant égales, non significatifs dans le cas de l'exposition élevée aux médicaments psychotropes. Cette exposition est associée à la présence d'au moins un des troubles mentaux évalués ( $\mathrm{RC}=6,12$; IC95\%: 3,70-10,30). 
TABLEAU 6 Caractéristiques des participants présentant un diagnostic de trouble mental

\begin{tabular}{lll}
\hline Caractéristiques & $\begin{array}{l}\text { Absence de } \\
\text { trouble mental } \\
\text { Fréquence }(\%)\end{array}$ & $\begin{array}{l}\text { Un ou l'autre des } \\
\text { troubles mentaux } \\
\text { Fréquence }(\%)\end{array}$ \\
\hline $\begin{array}{l}\text { Type de trouble } \\
\text { Humeur }\end{array}$ & $0(0)$ & $229(77,4)$ \\
$\quad$ Anxieux & $0(0)$ & $131(44,6)$ \\
$\begin{array}{l}\text { Toxicomanie } \\
\text { Dépendance à l'alcool }\end{array}$ & $59(3,0)$ & $35(11,8)$ \\
Dépendance aux drogues & $40(2,1)$ & $32(10,8)$ \\
Âge & & \\
15-24 & $331(16,8)$ & $41(13,4)$ \\
$25-44$ & $803(40,8)$ & $140(45,9)$ \\
$45+$ & $834(42,4)$ & $124(40,7)$ \\
État matrimonial & & $102(33,3)$ \\
Vivant avec un partenaire & $935(47,6)$ & $142(46,4)$ \\
Célibataire & $718(36,6)$ & $62(20,3)$ \\
Séparé/veuf/divorcé & $311(15,8)$ & $95(31,9)$ \\
En emploi, durant les 12 derniers mois & & $203(68,1)$ \\
Non & $380(19,4)$ & \\
Oui & $1580(80,6)$ &
\end{tabular}

Note: Les pourcentages sont calculés à l'intérieur des catégories de présence d'un trouble mental.

En somme, les personnes qui souffrent d'un trouble mental présentent des caractéristiques cliniques et sociodémographiques particulières (tableau 6). Elles présentent surtout un diagnostic de trouble de l'humeur (77,4\%), une dépendance à l'alcool $(11,8 \%)$ ou aux drogues $(10,8 \%)$. Célibataires $(46,4 \%)$, elles sont âgées de 25 à 44 ans $(45,9 \%)$ et n'ont pas occupé un emploi au cours des 12 derniers mois précédant l'enquête (31,9\%).

\section{Discussion}

Cette étude épidémiologique, menée auprès de 2433 personnes du territoire du sud-ouest de Montréal, a permis d'identifier certains facteurs sociodémographiques et diagnostics associés à l'utilisation des médicaments psychotropes.

Par ailleurs, la consommation d'au moins un médicament psychotrope s'est révélée plus élevée dans le sud-ouest de Montréal (20\%) que parmi la population québécoise, dans le cadre de l'enquête ESCC1.2 
(16\%). Le sud-ouest de Montréal est caractérisé par un indice de défavorisation très élevé $(6,2)$ comparativement aux autres régions de Montréal (Turpide \& Joseph, 2006). Le taux plus élevé de consommation de médicaments psychotropes observé pourrait être associé à ce niveau socioéconomique faible, comme cela a été évoqué récemment par une autre étude canadienne (Caron \& Liu, 2010). D’ailleurs, la présente étude a permis d'observer une association statistiquement significative entre la consommation de certains médicaments psychotropes, tels que les somnifères, les anxiolytiques et les antidépresseurs, et le fait d'être plus âgé, d'être une femme, une personne vivant seule, une personne ayant un niveau d'instruction peu élevé, un revenu faible et n'ayant pas occupé un emploi récemment. Ces résultats s'apparentent à ceux rapportés par l'ESCC (Enquête sur la santé dans les collectivités canadiennes du cycle 1.2, 2009) et d'autres recherches sur l'utilisation des médicaments psychotropes (Chien et al., 2008; Hohmann, 1989; Kaye et al., 2003; Trinkoff \& Anthony, 1990). De même, conformément aux résultats observés dans des recherches auprès d'adultes et de personnes âgées, les personnes célibataires, divorcées ou veuves sont aussi, dans la présente étude, plus exposées à des combinaisons de médicaments psychotropes en comparaison avec les personnes mariées (Cohidon et al., 2005; Empereur et al., 2003; Lesen et al., 2010).

En outre, la forte consommation de différents médicaments psychotropes dans le sud-ouest de Montréal est associée à une prévalence plus élevée de la dépendance à l'alcool ou aux drogues illicites, un taux nettement supérieur dans la zone épidémiologique étudiée $(6,3 \%)$ que dans l'échantillon québécois tiré de l'ESCC1.2 (1.9\%). Il s'y ajoute l'influence des forts taux de prévalence d'autres troubles mentaux tels que le trouble anxieux ou le trouble de l'humeur, puisque les personnes souffrant d'un trouble de santé mentale sont plus susceptibles de consommer des substances psychoactives (Kairouz et al., 2008). La prévalence annuelle des troubles de l'humeur rapportée dans la présente étude est d'ailleurs presque deux fois plus élevée que celle observée chez la population québécoise dans le cadre de l'enquête ESCC1.2 (5,9\% vs 3,6\%) (Kairouz et al., 2008).

Pour ce qui est de l'exposition élevée aux médicaments psychotropes, c'est-à-dire à une combinaison d'au moins trois types de ces médicaments, les résultats obtenus divergent de ceux auxquels on pouvait s'attendre. En effet, en présence des autres variables (âge, statut matrimonial, revenu du ménage, statut d'emploi et diagnostic de 
trouble mental), aucune différence statistiquement significative n'a été observée entre les hommes et les femmes. Ces résultats ne concordent pas avec ceux d'autres recherches qui ont révélé que la probabilité d'utilisation de trois médicaments psychotropes ou plus était plus élevée chez les femmes (Johnell et al., 2007; Lesen et al., 2010). De telles études ayant été menées chez des personnes âgées, on peut supposer qu'elles ne reflètent pas nécessairement la réalité d'un échantillon plutôt hétérogène incluant des personnes plus jeunes. Ces mêmes personnes âgées sont plus susceptibles de consommer plusieurs médicaments psychotropes; ce qui converge avec la recherche effectuée par Lesen et ses collaborateurs (2010). L'exposition élevée aux médicaments psychotropes selon l'âge est particulièrement préoccupante étant donné que les personnes âgées sont non seulement plus susceptibles de prendre plusieurs autres médicaments, mais aussi elles connaissent un plus grand risque d'effets indésirables liés à l'utilisation de somnifères, y compris les chutes, les fractures et les problèmes cognitifs (Glass et al., 2005). L'isolement social et familial apparaît comme un facteur potentiellement explicatif de cette polyconsommation (Allard et al., 1997).

En ce qui a trait à l'association entre le revenu et l'utilisation des médicaments psychotropes, elle varie selon les études (Blanc \& Marchand, 2010; Enquête sur la santé dans les collectivités canadiennes du cycle 1.2, 2009). Dans la présente recherche, une association négative statistiquement significative est rapportée pour cinq des six classes de médicaments lorsqu'on analyse les liens entre leur utilisation et le revenu du ménage (dans les analyses bivariées). Ces résultats correspondent à ceux rapportés dans l'étude ESCC1.2 et semblent conforter l'hypothèse qu'un revenu élevé peut favoriser une diminution du stress chez un individu et diminuer son besoin de recourir à la consommation de psychotropes. Cette hypothèse mériterait toutefois d'être vérifiée dans des études plus approfondies. Par contre, l'exposition élevée aux médicaments psychotropes n'est pas significativement associée au revenu lorsque celui-ci est en présence des autres variables dans le modèle multivarié. Ces résultats ne concordent pas avec ceux rapportés par Lesen et ses collègues (2010), qui indiquent que les personnes à faible revenu sont plus susceptibles d'utiliser trois médicaments psychotropes ou plus par rapport à celles dont le revenu est plus élevé. Toutefois, leur étude a été réalisée chez des personnes âgées de 75 ans et plus, dont la santé est reconnue pour être plus fragile que la population plus jeune, de même que le statut socioéconomique est 
connu pour être plus précaire (Lesen et al., 2010). L'interaction entre ces facteurs pourrait expliquer les différences entre les résultats observés dans cette population et ceux rapportés dans la présente étude.

Toujours concernant les déterminants socioéconomiques, les résultats rappellent tout de même que les personnes sans travail sont plus exposées aux médicaments psychotropes. Ils concordent avec ceux d'autres recherches du domaine qui ont montré que l'insécurité d'emploi ou même le fait d'occuper un emploi temporaire augmenteraient le risque de consommation de ces médicaments (Kivimaki et al., 2007). Une interprétation possible de ces résultats est que l'insécurité d'emploi réduirait le sentiment de contrôle sur l'environnement des personnes concernées et que cet aspect psychologique pourrait exercer à son tour des conséquences néfastes sur leur santé mentale (McDonough, 2000). D'ailleurs, il convient de rappeler que les résultats de la présente étude indiquent que la polyconsommation de médicaments psychotropes est associée à la présence d'un diagnostic de trouble mental, conformément à ceux d'une étude nationale menée aux États-Unis (Mojtabai \& Olfson, 2010). Comme le montrent les résultats complémentaires (tableau 6), une plus grande proportion de personnes aux prises avec des troubles mentaux sont aux prises avec l'isolement social, un facteur qui apparaît jouer un rôle déterminant dans l'exposition élevée aux médicaments psychotropes, tel que suggéré par l'étude d'Allard et ses collègues (1997).

\section{Considérations méthodologiques}

Il importe de noter ici certaines limites se rapportant à l'étude. La première concerne la mesure du type de médicament psychotrope consommé. Les questions ont été formulées de manière à ce que les répondants classent eux-mêmes leurs médicaments dans les catégories somnifères, régulateurs de l'humeur, anxiolytiques, antidépresseurs, antipsychotiques ou stimulants. Cette procédure présente des risques d'erreurs non seulement parce que les données recueillies reposent sur la classification effectuée par les participants mais aussi parce que l'information n'intègre pas le nombre de médicaments psychotropes différents au sein de chacune des classes. Comme des études récentes démontrent une tendance à prescrire plus d'un antidépresseur ou antipsychotique pour des troubles mentaux, cela pourrait amplifier l'erreur attribuable au jugement des participants (Glezer et al., 2009; Mojtabai \& Olfson, 2010). Dans les études futures, il serait donc opportun de demander plutôt aux répondants de nommer les médicaments 
psychotropes qu'ils consomment afin de minimiser les erreurs de classification. Toutefois, bien qu'il soit difficile d'évaluer l'imprécision reliée à l'identification des classes de médicaments dans la présente étude, l'Enquête Santé-Québec de 1987 peut fournir un ordre de grandeur. On y rapportait que $16 \%$ des médicaments avaient été faussement rapportés par les répondants comme des tranquillisants, sédatifs ou somnifères (Laurier et al., 1990). Il y aurait donc lieu de croire que la majorité des médicaments rapportés par les participants de la présente étude ont été classés correctement, d'autant plus que le questionnaire qui a été administré suggérait quelques noms de médicaments appartenant aux différentes classes étudiées, pour faciliter la tâche d'identification. Toutefois, certains médicaments sont prescrits comme antipsychotique, anxiolytique ou antidépresseur, comme la quétiapine (seroquel) dont les effets cliniques varient selon le dosage et les catégories de diagnostics traités (Zhornistky et al., 2011).

Une autre limite méthodologique est celle reliée au taux de participation (48,7\%), qui demeure modeste, bien qu'il s'avère plus élevé que le taux médian rapporté dans une méta-analyse d'études épidémiologiques (Morton et al., 2006). Ce taux de réponse pourrait entraîner une sous-représentation des personnes les plus vulnérables, généralement les plus difficiles à recruter dans ce genre d'étude, la participation étant notamment affectée par l'histoire familiale et médicale des personnes éligibles contactées (Morton et al., 2006). Ainsi, la proportion de personnes qui consomment trois médicaments psychotropes ou plus serait sous-estimée dans cette étude.

Par ailleurs, la taille de l'échantillon, dans une étude de ce type, ne permet pas de ventiler les résultats en fonction d'un grand nombre de catégories si les taux de prévalence sont faibles. Ainsi, parmi les 2433 personnes ayant répondu au questionnaire, seules 75 ont consommé trois psychotropes ou plus. Cette relative faiblesse de la taille du souséchantillon à l'étude ainsi que le déséquilibre qui en résulte limitent possiblement la découverte de relations statistiquement significatives. Une mesure d'exposition reposant sur la consommation d'au moins un ou deux psychotropes offrirait davantage de possibilités à cet effet.

Enfin, une autre limite de l'étude qu'il convient de noter est attribuable à sa nature transversale: on y mesure au même moment l'usage des médicaments psychotropes et la présence des facteurs qui y sont associés. Si l'âge, le statut matrimonial, le statut d'emploi et la présence d'un trouble mental contribuent à expliquer le risque d'une polyconsommation, il est difficile en revanche de conclure à l'existence de liens 
de causalité entre ces phénomènes. Il serait intéressant donc de mener une étude longitudinale afin de situer temporellement l'exposition à des facteurs sociodémographiques et cliniques et voir comment ces facteurs influent sur le changement dans l'usage des psychotropes en termes d'incidence ou de persistance.

\section{Conclusion}

La présente étude a permis d'examiner les facteurs associés à l'exposition à une combinaison de médicaments psychotropes auprès d'une population adulte du Québec. Les personnes âgées, séparées, vivant seules, n'ayant pas d'emploi, et surtout celles qui présentent au moins un trouble mental sont davantage exposées à la consommation de trois médicaments psychotropes ou plus. Les études futures permettront de déterminer la part de variation respectivement attribuable aux caractéristiques sociodémographiques et au diagnostic. Il convient de vérifier, dans des études futures, si les troubles mentaux et la détresse ne jouent pas un effet médiateur dans les associations entre les caractéristiques sociodémographiques et l'exposition aux médicaments psychotropes. Il sera aussi opportun d'étudier les combinaisons de médicaments psychotropes le plus souvent associées à une exposition concomitante élevée. Il importe d'identifier les classes de médicaments en cause, afin de prévenir une utilisation inappropriée, voire à risque de ceux-ci.

Les combinaisons de différentes classes de médicaments psychotropes ne présentent pas toutes une efficacité démontrée (Mojtabai \& Olfson, 2010). Aussi les présents résultats suggèrent la nécessité de considérer l'isolement social et la prévalence des troubles mentaux pour contextualiser l'exposition élevée aux médicaments psychotropes. La polypharmacie peut en effet présenter des risques importants si elle ne s'inscrit pas dans la logique d'un protocole d'intervention clinique cohérent.

\section{REMERCIEMENTS}

Cette recherche a été menée en partie grâce au soutien financier des Instituts de recherche en santé du Canada (IRSC, CTP - 79839). Des remerciements sont aussi adressés à tous ceux qui ont généreusement accepté de participer à cette étude ainsi qu'à la statisticienne du projet, Mme Aihua Liu, pour son assistance et ses conseils au cours des analyses. 


\section{RÉFÉRENCES}

Alessi-Severini, S., Biscontri, R. G., Collins, D. M., Kozyrskyj, A., Sareen, J., \& Enns, M. W. (2008). Utilization and costs of antipsychotic agents : a Canadian population-based study, 1996-2006, Psychiatr Serv, 59(5), 547-553.

Alla, F., \& Baumann, M. (2003). 5 year trajectories for dependence to psychotropics in a cohort of consumers, Therapie, 58(2), 145-151.

Allard, J., Allaire, D., Leclerc, G., \& Langlois, S.-P. (1997). L'influence des relations familiales et sociales sur la consommation de médicaments psychotropes chez les personnes âgées, Santé mentale au Québec, 22(1), 164-182.

Beck, C. A., Williams, J. V., Wang, J. L., Kassam, A., El-Guebaly, N., Currie, S. R., Patten, S. B., 2005, Psychotropic medication use in Canada, Can J Psychiatry, 50(10), 605-613.

Blanc, M. E., \& Marchand, A. (2010). The contribution of work and other social determinants to the onset of psychotropic drug use among workers in Canada, Can J Public Health, 101 Suppl 1, S63-68.

Caron, J., Fleury, M. J., Perreault, M., Crocker, A., Tremblay, J., Tousignant, M., Daniel, M. (2012). Prevalence of psychological distress and mental disorders, and use of mental health services in the epidemiological catchment area of Montreal South-West, BMC Psychiatry, 12(1), 183.

Caron, J., \& Liu, A. (2010). A descriptive Study on the Prevalence of Psychological Distress and Mental Disorders in the Canadian Population: Comparaison between Low-income and Non Low-income Populations, Chronic Diseases in Canada, 30(3), 84-94.

Chien, I. C., Hsu, J. H., Bih, S. H., Lin, C. H., Chou, Y. J., Lee, C. H., \& Chou, P. (2008). Prevalence, correlates, and disease patterns of antipsychotic use in Taiwan, Psychiatry Clin Neurosci, 62(6), 677-684.

Cohidon, C., Alla, F., Chau, N., \& Michaely, J. P. (2005). Tobacco, alcohol and psychotropic drugs in Lorraine, epidemiological survey of the general population, Sante Publique, 17(3), 325-338.

Comité permanent de lutte à la toxicomanie (2003). La consommation de psychotropes: portrait et tendances au Québec. Retrieved from http://www.stat. gouv.qc.ca/publications/sante/troubles_mentaux.htm (consulté le 10 août 2012)

Empereur, F., Baumann, M., Alla, F., \& Briancon, S. (2003). Factors associated with the consumption of psychotropic drugs in a cohort of men and women aged 50 and over, J Clin Pharm Ther, 28(1), 61-68.

Enquête nationale sur la santé de la population (ENSP) (2003-2005), from http:// www.hc-sc.gc.ca/fn-an/surveill/nutrition/population/index-fra.php (consulté le 15 septembre 2011)

Enquête sur la santé dans les collectivités canadiennes (cycle 1.2), (2009). Utilisation de services et consommation de médicaments liées aux problèmes de santé mentale chez les adultes québécois, from http://www.stat.gouv.qc.ca/ publications/sante/pdf2009/medicaments_sante_mentale.pdf (consulté le 30 septembre 2011) 
Essock, S., Covell, N., Leckman-Westin, E., Lieberman, J., Sederer, L., Kealey, E., \& Finnerty, M. (2009). Identifying clinically questionable psychotropic prescribing practices for Medicaid recipients in New York State, Psychiatric Services, 60(12), 1595-1602.

Glass, J., Lanctôt, K. L., Herrmann, N., Sproule, B. A., \& Busto, U. E. (2005). Sedative hypnotics in older people with insomnia: meta-analysis of risks and benefits, Bmj, 331(7526), 1169.

Glezer, A., Byatt, N., Cook Jr, R., \& Rothschild, A. J. (2009). Polypharmacy prevalence rates in the treatment of unipolar depression in an outpatient clinic, Journal of affective disorders, 117(1), 18-23.

Hall, W. D., Mant, A., Mitchell, P. B., Rendle, V. A., Hickie, I. B., \& McManus, P. (2003). Association between antidepressant prescribing and suicide in Australia, 1991-2000: trend analysis, BMJ, 326(7397), 1008.

Hemels, M. E., Koren, G., \& Einarson, T. R. (2002). Increased use of antidepressants in Canada: 1981-2000, Ann Pharmacother, 36(9), 1375-1379.

Hohmann, A. A. (1989). Gender bias in psychotropic drug prescribing in primary care, Med Care, 27(5), 478-490.

Hollingworth, S. A., Burgess, P. M., \& Whiteford, H. A. (2003). Affective and anxiety disorders : prevalence, treatment and antidepressant medication use, Aust N Z J Psychiatry, 44(6), 513-519.

Johnell, K., Fastbom, J., Rosen, M., \& Leimanis, A. (2007). Innapropriate drug use in the elderly: a nationwide register-based study, Ann Pharmacother, 41(7), 1243-1248.

Kairouz, S., Boyer, R., Nadeau, L., Perreault, M., \& Fiset-Laniel, J. (2008) Troubles mentaux, toxicomanie et autres problèmes liés à la santé mentale chez les adultes québécois. Enquête sur la santé dans les collectivités canadiennes (cycle 1.2), from www.stat.gouv.qc.ca/publications/sante/troubles_mentaux. htm (consulté le 10 juillet 2012)

Kaye, J. A., Bradbury, B. D., \& Jick, H. (2003). Changes in antipsychotic drug prescribing by general practitioners in the United Kingdom from 1991 to 2000 : a population-based observational study, Br J Clin Pharmacol, 56(5), 569-575.

Kessler, R. C., Wittchen, H. U., Abelson, J. M., McGonagle, K., Schwarz, N., Kendler, K. S., Zhao, S. (1998). Methodological studies of the Composite International Diagnostic Interview (CIDI) in the US National Comorbidity Survey (NCS), International Journal of Methods in Psychiatric Research, 7(4), 210-211.

Kivimaki, M., Honkonen, T., Wahlbeck, K., Elovainio, M., Pentti, J., Klaukka, T., Vahtera, J., 2007, Organisational downsizing and increased use of psychotropic drugs among employees who remain in employment, $J$ Epidemiol Community Health, 61(2), 154-158.

Klarin, I., Wimo, A., \& Fastbom, J. (2005). The association of inappropriate drug use with hospitalization and mortality: a population-based study of the very old, Drugs Aging, 22(1), 69-82. 
Lau, D. T., Kasper, J. D., Potter, D. E., Lyles, A., \& Bennett, R. G. (2005). Hospitalization and death associated with potentially inappropriate medication prescriptions among elderly nursing home residents, Arch Intern Med, 165(1), 68-74.

Laurier, C., Dumas, J., Grégoire, J.-P., \& Duvak, L. (1990). L'utilisation des tranquillisants, sédatifs et somnifères: analyse des données de l'enquête Santé Québec 1987. Direction des communications, ministère de la Santé et des Services sociaux: Gouvernement du Québec.

Lesen, E., Andersson, K., Petzold, M., \& Carlsten, A. (2010). Socioeconomic determinants of psychotropic drug utilisation among elderly: a national population-based cross-sectional study, BMC Public Health, 10, 118.

McDonough, P., 2000, Job insecurity and health, Int J Health Serv, 30(3), 453-476.

Mojtabai, R., \& Olfson, M. (2010). National trends in psychotropic medication polypharmacy in office-based psychiatry, Archives of General Psychiatry, 67(1), 26-36.

Morton, L. M., Cahill, J., \& Hartge, P. (2006). Reporting participation in epidemiologic studies: a survey of practice, Am J Epidemiol, 163, 197-203.

Patrick, V., Levin, E., \& Schlieffer, S. (2005). Antipsychotic polypharmacy: is there evidence for its use?, Journal of Psychiatric Practice, 11(4), 248-257.

Percudani, M., Barbui, C., Fortino, I., \& Petrovich, L. (2005). Epidemiology of first- and second-generation antipsychotic agents in Lombardy, Italy, Pharmacopsychiatry, 38(3), 128-131.

Perri, M., Menon, A. M., Deshpande, A. D., Shinde, S. B., Jiang, R., Cooper, J. W., Cook, C. L., Griffin, S. C., Lorys, R. A. (2005). Adverse outcomes associated with inappropriate drug use in nursing homes, Ann Pharmacother, 39(3), 405-411.

Preskorn, S. H., \& Lacey, R. L. (2007). Polypharmacy: when is it rational?, Journal of Psychiatric Practice, 13(2), 97-105.

Reseland, S., Bray, I., \& Gunnell, D. (2006). Relationship between antidepressant sales and secular trends in suicide rates in the Nordic countries, Br J Psychiatry, 188, 354-358.

Trinkoff, A. M., \& Anthony, J. C. (1990). Gender differences in initiation of psychotherapeutic medicine use, Acta Psychiatr Scand, 81(1), 32-38.

Turpide, B., \& Joseph, G. (2006). Portrait sociodémographique, socioéconomique et scolaire de la région de Montréal, from http://www.centre123go.ca/upload/ documents/62A2B479-2DA8-0507-B71E-33334FA113D1-portrait_montreal_ QEF.pdf (consulté le 12 septembre 2011)

Zhornitsky, S., Potvin, S., Moteshafi, H., Dubreucq, S., Rompré, P. P., \& Stip, E. (2011) Dose-response and comparative efficacy and tolerability of quetiapine across psychiatric disorders: a systematic review of the placebo-controlled monotherapy and add-on trials, Int Clin Psychopharmacol, 26(4), 183-92. 